\title{
A new analysis of the MiniBooNE low-energy excess
}

\section{Giunti, ${ }^{a}$ A. loannisian ${ }^{b, c, d}$ and G. Ranucci ${ }^{e}$}

\author{
${ }^{a}$ Istituto Nazionale di Fisica Nucleare (INFN), Sezione di Torino, \\ Via P. Giuria 1, I-10125 Torino, Italy \\ ${ }^{b}$ CERN, Theory Division, \\ CH-1211 Geneva 23, Switzerland \\ ${ }^{c}$ Yerevan Physics Institute, \\ Alikhanian Brothers 2, Yerevan-36, Armenia \\ ${ }^{d}$ Institute for Theoretical Physics and Modeling, \\ Yerevan-36, Armenia \\ ${ }^{e}$ Istituto Nazionale di Fisica Nucleare (INFN), Sezione di Milano, \\ I-20133 Milano, Italy \\ E-mail: carlo.giunti@to.infn.it, ara.ioannisyan@cern.ch, \\ gioacchino.ranucci@mi.infn.it
}

ABSTRACT: We present the results of a new analysis of the data of the MiniBooNE experiment taking into account the additional background of photons from $\Delta^{+/ 0}$ decay proposed in ref. [1] and additional contributions due to coherent photon emission, incoherent production of higher mass resonances, and incoherent non-resonant nucleon production. We show that the new background can explain part of the MiniBooNE low-energy excess and the statistical significance of the MiniBooNE indication in favor of short-baseline neutrino oscillation decreases from $5.1 \sigma$ to $3.6 \sigma$. We also consider the implications for short-baseline neutrino oscillations in the $3+1$ active-sterile neutrino mixing framework. We show that the new analysis of the MiniBooNE data indicates smaller active-sterile neutrino mixing and may lead us towards a solution of the appearance-disappearance tension in the global fit of short-baseline neutrino oscillation data.

Keywords: Beyond Standard Model, Neutrino Physics

ArXiv EPrint: 1912.01524 


\section{Contents}

1 Introduction 1

2 The MiniBooNE single- $\gamma$ background 1

3 Short-baseline neutrino oscillations 5

$\begin{array}{llr}4 & \text { Conclusions } & 9\end{array}$

\section{Introduction}

The MiniBooNE experiment [2] found a significant excess of low-energy $\nu_{e}$-like events that could be due to short-baseline $\nu_{\mu} \rightarrow \nu_{e}$ oscillations generated by active-sterile neutrino mixing [3-5] or to other physics beyond the Standard Model [6-9]. However, the oscillation explanation of the MiniBooNE low-energy excess is in tension with the data of other shortbaseline neutrino oscillation experiments [10-12] and the non-oscillatory explanations are disfavored by other measurements [13]. A possible solution of this conundrum lies in a reevaluation of the estimated background that can decrease the low-energy excess. Among the different sources of background an important one is the single- $\gamma$ background that cannot be distinguished from $\nu_{e}$-like events in the MiniBooNE detector.

The MiniBooNE single- $\gamma$ background was studied theoretically in ref. [14], where it was found that it is a factor of about 2 larger than that estimated by the MiniBooNE collaboration. On the other hand, the later theoretical studies in refs. [15, 16] found an approximate agreement with the MiniBooNE estimate. However, doubts on the real value of the MiniBooNE single- $\gamma$ background still remain. They motivated the ongoing investigation in the MicroBooNE experiment at Fermilab [17], which is able to distinguish between photon and $\nu_{e}$ events by using a Liquid Argon Time Projection Chamber (LArTPC). If the MicroBooNE experiment finds an excess of single- $\gamma$ events that can explain the MiniBooNE low-energy excess, it will be necessary to understand its origin.

In this paper we propose an increase of the estimated MiniBooNE single- $\gamma$ background that can explain part of the MiniBooNE low-energy excess. In section 2 we present the reasons of the increase of the MiniBooNE single- $\gamma$ background and we calculate the enhancement. In section 3 we discuss the implications for short-baseline active-sterile oscillations, and in section 4 we draw our conclusions.

\section{The MiniBooNE single- $\gamma$ background}

In MiniBooNE the single- $\gamma$ background is thought to be due mainly to $\Delta \rightarrow N \gamma$ photons produced by the decay $\Delta^{+/ 0} \rightarrow p / n+\gamma$ of $\Delta^{+/ 0}$ 's produced in neutral-current $\nu_{\mu}$ 
interactions with the mineral oil $\left(\mathrm{CH}_{2}\right)$ of the detector. The MiniBooNE collaboration estimated this background through the measurement of $\pi^{0}$ 's that are produced by the decay $\Delta^{+/ 0} \rightarrow p / n+\pi^{0}$, using the branching fractions $[18,19]$

$$
\begin{aligned}
& \operatorname{Br}\left(\Delta^{+/ 0} \rightarrow p / n+\gamma\right)=(6.0 \pm 0.5) \times 10^{-3}, \\
& \operatorname{Br}\left(\Delta^{+/ 0} \rightarrow p / n+\pi^{0}\right) \simeq 2 / 3
\end{aligned}
$$

Final state interactions (FSI) cause the absorption of a fraction of the $\pi^{0}$ 's in the carbon nucleus that was estimated to be about $37.5 \%$ by the MiniBooNE collaboration [20]. ${ }^{1}$ However, in ref. [1] one of us noted that measurements of $\pi^{0}$ photoproduction on nuclei [21, 22] indicate that the fraction of $\pi^{0}$ 's that emerge from the nucleus and can be observed is given by

$$
\frac{N_{\pi^{0}}^{\mathrm{FSI}}}{N_{\pi^{0}}^{0}}=\frac{\sigma_{\mathrm{FSI}}\left(\gamma+{ }^{A} \mathcal{N} \rightarrow \pi^{0}+X\right)}{\sigma_{0}\left(\gamma+{ }^{A} \mathcal{N} \rightarrow \pi^{0}+X\right)} \simeq \frac{A^{2 / 3}}{A}=A^{-1 / 3},
$$

where $A$ is the mass number of the target nucleus ${ }^{A} \mathcal{N}, \sigma_{\text {FSI }}$ denotes the measured cross section which includes final state interactions and $\sigma_{0}$ denotes the theoretical cross section without final state interactions. Since the nuclear radius scales approximately as $A^{1 / 3}$, the $A^{2 / 3}$ dependency of $\sigma_{\mathrm{FSI}}\left(\gamma+{ }^{A} \mathcal{N} \rightarrow \pi^{0}+X\right)$ indicates that only the nuclear surface contributes, whereas all the $\pi^{0}$ that are produced in the nuclear interior are absorbed [23]. However, the uncertainties of the $A^{2 / 3}$ scaling in eq. (2.3) are not known.

Our proposal is to consider the extreme total absorption of the $\pi^{0}$ that are produced in the nuclear interior as indicated by the $\pi^{0}$ photoproduction data [21, 22] as the possible cause of an increase of the estimated MiniBooNE single- $\gamma$ background that can explain at least part of the MiniBooNE low-energy excess. In other words, we consider eq. (2.3) as an ansatz of the effects of FSI $\pi^{0}$ absorption in a nucleus that is motivated by the photoproduction data. The resulting estimate of the probability of $\pi^{0}$ escape from the ${ }^{12} \mathrm{C}$ nucleus is

$$
\widetilde{S}_{\mathrm{C}}\left(\pi^{0}\right) \simeq 12^{-1 / 3}=0.437
$$

that is smaller than that estimated by the MiniBooNE collaboration [20],

$$
S_{\mathrm{C}}^{\mathrm{MB}}\left(\pi^{0}\right)=0.625
$$

According to our estimation, the number of $\Delta^{+/ 0}$ produced in neutral-current $\nu_{\mu}$ interactions with ${ }^{12} \mathrm{C}$ and the number of $\gamma^{\prime}$ s generated by their decay is a factor $\left[\widetilde{S}_{\mathrm{C}}\left(\pi^{0}\right)\right]^{-1} \simeq 2.3$ larger than that obtained from the measurement of $\pi^{0}$ 's without taking into account FSI. This enhancement of the $\Delta \rightarrow N \gamma$ background due to $\pi^{0}$ FSI in the ${ }^{12} \mathrm{C}$ nucleus is in approximate agreement with the theoretical estimation of a factor about 2.4 in ref. [24] and it is larger than the factor $\left[S_{\mathrm{C}}^{\mathrm{MB}}\left(\pi^{0}\right)\right]^{-1}=1.6$ considered by the MiniBooNE collaboration [20].

\footnotetext{
${ }^{1}$ In this paper we do not consider the new MiniBooNE data presented in ref. [20] because there is still no available data release.
} 
Moreover, the MiniBooNE collaboration assumed that "single gamma events are assumed to come entirely from $\Delta$ radiative decay" [20], neglecting the additional contributions to $\gamma$ production from coherent photon emission, incoherent production of higher mass resonances, and incoherent non-resonant nucleon production [15, 16]. Taking into account these contributions and our value (2.4) of the probability of $\pi^{0}$ escape from the ${ }^{12} \mathrm{C}$ nucleus, the ratio of single- $\gamma$ events to $\mathrm{NC} \pi^{0}$ events is given by

$$
\widetilde{R}=\frac{N_{\mathrm{H}}^{\mathrm{th}}(\Delta \rightarrow N \gamma)+N_{\mathrm{C}}^{\mathrm{th}}(\Delta \rightarrow N \gamma)+N_{\mathrm{coh}}^{\mathrm{th}}(\gamma)+N^{\mathrm{th}}\left(N^{*} \rightarrow N \gamma\right)+N^{\mathrm{th}}(N \rightarrow N \gamma)}{\widetilde{N}_{\mathrm{tot}}^{\mathrm{th}, \mathrm{obs}}\left(\pi^{0}\right)}
$$

The contributions in the numerator are, respectively, the theoretically predicted numbers of single- $\gamma$ events due to $\Delta \rightarrow N \gamma$ in $\mathrm{H}, \Delta \rightarrow N \gamma$ in C, coherent photon emission, incoherent production of higher mass resonances $\left(N^{*} \rightarrow N \gamma\right)$ and incoherent non-resonant nucleon production $(N \rightarrow N \gamma)$. The denominator is the theoretically predicted total number of observed $\pi^{0}$ events. Note that only the denominator of eq. (2.6) depends on the probability of $\pi^{0}$ escape from the ${ }^{12} \mathrm{C}$ nucleus, because a larger escape probability implies a larger number of observed $\pi^{0}$ events. The tilde notation indicates that $\widetilde{N}_{\text {tot }}^{\text {th,obs }}\left(\pi^{0}\right)$ corresponds to our value $\widetilde{S}_{\mathrm{C}}\left(\pi^{0}\right)$ in eq. (2.4) of such probability.

We can write eq. (2.6) as

$$
\begin{aligned}
\widetilde{R}= & \frac{N_{\mathrm{H}}^{\mathrm{th}}(\Delta \rightarrow N \gamma)+N_{\mathrm{C}}^{\mathrm{th}}(\Delta \rightarrow N \gamma)}{N_{\mathrm{tot}}^{\mathrm{th}, \mathrm{obs}}\left(\pi^{0}\right)}\left(\frac{N_{\mathrm{tot}}^{\mathrm{th}, \mathrm{obs}}\left(\pi^{0}\right)}{\widetilde{N}_{\mathrm{tot}}^{\mathrm{th}, \mathrm{obs}}\left(\pi^{0}\right)}\right) \\
& \times\left(1+f_{\mathrm{coh}}^{\mathrm{th}}+f_{N^{*}}^{\mathrm{th}}+f_{N}^{\mathrm{th}}\right),
\end{aligned}
$$

where $N_{\text {tot }}^{\text {th,obs }}\left(\pi^{0}\right)$ is the total number of observed $\pi^{0}$ events estimated by the MiniBooNE collaboration using the probability of $\pi^{0}$ escape from the ${ }^{12} \mathrm{C}$ nucleus $S_{\mathrm{C}}^{\mathrm{MB}}\left(\pi^{0}\right)$ in eq. (2.5). In eq. (2.7) $f_{\mathrm{coh}}^{\text {th }}, f_{N^{*}}^{\text {th }}$, and $f_{N}^{\text {th }}$ are, respectively, the theoretically predicted ratios of $\gamma^{\prime} \mathrm{s}$ generated coherently, by higher mass resonances, and non-resonant nucleon production with respect to those generated by $\Delta$ decay. In this way, we separated these contributions from the those generated by $\Delta$ decay that were considered by the MiniBooNE collaboration.

The first fraction on the right-hand side of eq. (2.7) is the ratio of single- $\gamma$ events to NC $\pi^{0}$ events estimated by the MiniBooNE collaboration:

$$
R_{\mathrm{MB}}=\frac{N_{\mathrm{H}}^{\mathrm{th}}(\Delta \rightarrow N \gamma)+N_{\mathrm{C}}^{\mathrm{th}}(\Delta \rightarrow N \gamma)}{N_{\mathrm{tot}}^{\mathrm{th}, \mathrm{obs}}\left(\pi^{0}\right)}=0.0091
$$

The second fraction on the right-hand side of eq. (2.7) can be calculated by writing it as

$$
\frac{N_{\text {tot }}^{\text {th,obs }}\left(\pi^{0}\right)}{\widetilde{N}_{\text {tot }}^{\text {th,obs }}\left(\pi^{0}\right)}=\frac{N_{\text {abs }}^{\text {th,obs }}\left(\pi^{0}\right)+N_{\text {noabs }}^{\text {th,obs }}\left(\pi^{0}\right)}{\widetilde{N}_{\text {abs }}^{\text {th,obs }}\left(\pi^{0}\right)+N_{\text {noabs }}^{\text {th,obs }}\left(\pi^{0}\right)}
$$

where $N_{\text {abs }}^{\text {th,obs }}\left(\pi^{0}\right)$ and $\widetilde{N}_{\text {abs }}^{\text {th,obs }}\left(\pi^{0}\right)$ are the theoretically predicted numbers of observed $\pi^{0}$ produced in processes with absorption of $\pi^{0}$ in the C nucleus, whereas $N_{\text {noabs }}^{\text {th,obs }}\left(\pi^{0}\right)$ is the 


\begin{tabular}{|c|ccc|ccc|}
\hline & \multicolumn{3}{|c|}{$\nu$ mode } & \multicolumn{3}{c|}{$\bar{\nu}$ mode } \\
$E_{\nu}^{\mathrm{QE}}(\mathrm{GeV})$ & {$[0.2,0.3]$} & {$[0.3,0.475]$} & {$[0.475,1.3]$} & {$[0.2,0.3]$} & {$[0.3,0.475]$} & {$[0.475,1.3]$} \\
\hline$f_{\mathrm{coh}}^{\text {th }}$ & 0.09 & 0.13 & 0.06 & 0.16 & 0.16 & 0.07 \\
$f_{N^{*}}^{\text {th }}$ & 0.02 & 0.02 & 0.13 & 0.03 & 0.02 & 0.13 \\
$\widetilde{R} / R_{\mathrm{MB}}$ & 1.52 & 1.56 & 1.61 & 1.62 & 1.61 & 1.62 \\
\hline
\end{tabular}

Table 1. Estimations of $f_{\mathrm{coh}}^{\mathrm{th}}$ and $f_{N^{*}}^{\mathrm{th}}$ from table 2 of ref. [16] in three ranges of reconstructed neutrino energy $E_{\nu}^{\mathrm{QE}}$ in the $\nu$ and $\bar{\nu}$ modes of the MiniBooNE experiment, and the corresponding values of the enhancement factor $\widetilde{R} / R_{\mathrm{MB}}$ that we obtained considering $f_{N}^{\text {th }} \simeq 0.1$ [15] and our value (2.4) of the probability of $\pi^{0}$ escape from the ${ }^{12} \mathrm{C}$ nucleus.

theoretically predicted numbers of observed $\pi^{0}$ produced in processes without absorption of $\pi^{0}$ in the C nucleus. Note that only $N_{\text {abs }}^{\text {th,obs }}\left(\pi^{0}\right)$ and $\widetilde{N}_{\text {abs }}^{\text {thobs }}\left(\pi^{0}\right)$ depend on the probability of $\pi^{0}$ escape from the ${ }^{12} \mathrm{C}$ nucleus and are given by $N_{\mathrm{abs}}^{\mathrm{th}, \mathrm{obs}}\left(\pi^{0}\right)=N_{\mathrm{abs}}^{\mathrm{th}, \text { prod }}\left(\pi^{0}\right) S_{\mathrm{C}}^{\mathrm{th}}\left(\pi^{0}\right)$ and $\tilde{N}_{\text {abs }}^{\text {th,obs }}\left(\pi^{0}\right)=N_{\text {abs }}^{\text {th,prod }}\left(\pi^{0}\right) \widetilde{S}_{\mathrm{C}}^{\mathrm{th}}\left(\pi^{0}\right)$. Therefore, we can write eq. (2.9) as

$$
\frac{N_{\text {tho }}^{\text {th,obs }}\left(\pi^{0}\right)}{\widetilde{N}_{\text {tot }}^{\text {th,obs }}\left(\pi^{0}\right)}=\frac{1+\frac{N_{\text {noabs }}^{\text {th,obs }}\left(\pi^{0}\right)}{N_{\text {abs }}^{\text {th,obs }}\left(\pi^{0}\right)}}{\frac{\widetilde{S}_{\mathrm{C}}^{\text {th }}\left(\pi^{0}\right)}{S_{\mathrm{C}}^{\text {th }}\left(\pi^{0}\right)}+\frac{N_{\text {noabs }}^{\text {th,obs }}\left(\pi^{0}\right)}{N_{\text {abs }}^{\text {th,obs }}\left(\pi^{0}\right)}} .
$$

We obtained the value of $N_{\text {noabs }}^{\text {th,obs }}\left(\pi^{0}\right) / N_{\text {abs }}^{\text {th,obs }}\left(\pi^{0}\right)$ from the contributions to the MiniBooNE $\pi^{0}$ event sample given in ref. [20]:

$$
\frac{N_{\text {noabs }}^{\text {th,obs }}\left(\pi^{0}\right)}{N_{\text {abs }}^{\text {th,obs }}\left(\pi^{0}\right)} \simeq 0.496 .
$$

The resulting enhancement factor of the MiniBooNE single- $\gamma$ background is

$$
\frac{\widetilde{R}}{R_{\mathrm{MB}}} \simeq 1.25\left(1+f_{\mathrm{coh}}^{\mathrm{th}}+f_{N^{*}}^{\mathrm{th}}+f_{N}^{\mathrm{th}}\right)
$$

The authors of ref. [16] calculated the number of single photon events from neutral current interactions at MiniBooNE. From their table 2 we obtained the estimates of $f_{\text {coh }}^{\text {th }}$ and $f_{N^{*}}^{\text {th }}$ in table 1, considering three ranges of $E_{\nu}^{\mathrm{QE}}$ in the $\nu$ and $\bar{\nu}$ modes of the MiniBooNE experiment. For $f_{N}^{\text {th }}$ we considered the $10 \%$ value estimated in ref. [15]. Note that we did not use the absolute values of the events calculated in refs. $[15,16]$, that are in agreement with the MiniBooNE estimates, and hence in disagreement with our estimations. We used only the relative values of the $\gamma$ 's generated coherently, by higher mass resonances, and non-resonant nucleon production, whose estimation can be considered more accurate.

As shown in table 1 , we find an enhancement $\widetilde{R} / R_{\mathrm{MB}}$ of the single- $\gamma$ background in MiniBooNE by a factor between 1.52 and 1.62 depending on the energy range and neutrino or antineutrino mode of the MiniBooNE experiment. This increase of the single$\gamma$ background can explain in part the low-energy MiniBooNE excess, because its largest 
contribution occur in the lowest energy bins, as one can see from figures 1(a) and 1(b) that reproduce the MiniBooNE event histograms in neutrino and antineutrino mode in refs. [2, 25].

Figure 1 shows a comparison of the standard MiniBooNE event histograms (figures 1(a) and 1(b)) with those obtained with our reevaluation of the single- $\gamma$ background (figures 1(c) and 1(d)). One can see that in the reproductions 1(a) and 1(b) of the original MiniBooNE histograms the low-energy bins show a large excess with respect to the background prediction. The excess is significantly reduced with our enhanced single- $\gamma$ background. Only the first energy bin remains with a large visible excess.

The improvement of the fit of the MiniBooNE data is quantified by $\chi^{2} / \mathrm{NDF}=36.9 / 22$, corresponding to a goodness-of-fit of $2 \%$ obtained with the enhanced single- $\gamma$ background, compared to $\chi^{2} / \mathrm{NDF}=53.0 / 22$, corresponding to a goodness-of-fit of $0.02 \%$, obtained in the standard analysis of MiniBooNE data.

\section{Short-baseline neutrino oscillations}

The reevaluation of the low-energy MiniBooNE excess has important implications for the interpretation of the MiniBooNE data in terms of short-baseline neutrino oscillations due to active-sterile neutrino mixing. In the following we consider the $3+1$ scenario in which in addition to the three standard light massive neutrinos $\nu_{1}, \nu_{2}, \nu_{3}$, with respective masses $m_{1}, m_{3}, m_{3}$, there is a heavier neutrino $\nu_{4}$ with mass $m_{4}$. The masses of the three standard light massive neutrinos have small separations, determined by the measurements of solar, atmospheric and long-baseline oscillations: $\Delta m_{21}^{2} \simeq 7.4 \times 10^{-5} \mathrm{eV}^{2}$ and $\left|\Delta m_{31}^{2}\right| \simeq 2.5 \times$ $10^{-3} \mathrm{eV}^{2}$, with $\Delta m_{i j}^{2} \equiv m_{i}^{2}-m_{j}^{2}$. A much larger squared mass difference $\Delta m_{41}^{2} \simeq \Delta m_{42}^{2} \simeq$ $\Delta m_{43}^{2} \gtrsim 0.1 \mathrm{eV}^{2}$ can generate short-baseline $\stackrel{(-)}{\nu} \rightarrow \stackrel{(-)}{\nu}$ oscillations that may explain the LSND and MiniBooNE anomalies, as well as other indications of short-baseline neutrino oscillations [3-5]. The probability of short-baseline $\stackrel{(-)}{\nu} \rightarrow \stackrel{(-)}{\nu}$ oscillations is given by

$$
\underset{\substack{(-) \\ \nu_{\mu} \rightarrow \nu_{e}}}{\mathrm{SBL}}=\sin ^{2} 2 \vartheta_{e \mu} \sin ^{2}\left(\frac{\Delta m_{41}^{2} L}{4 E}\right)
$$

where $E$ is the neutrino energy, $L$ is the source-detector distance, and $\sin ^{2} 2 \vartheta_{e \mu}=$ $4\left|U_{e 4}\right|^{2}\left|U_{\mu 4}\right|^{2}$, where $U$ is the $4 \times 4$ unitary mixing matrix.

Figure 2 shows a comparison of the standard allowed regions in the $\left(\sin ^{2} 2 \vartheta_{e \mu}, \Delta m_{41}^{2}\right)$ plane obtained from the analysis of the MiniBooNE data and those obtained with our enhanced single- $\gamma$ background. The goodness-of-fit and the best-fit values of the oscillation parameters are listed in table 2 , and the best fit event histograms are shown in figure 1 . The difference between the $\chi_{\min }^{2}$ with oscillations and the $\chi^{2}$ without oscillations is 30.2 and 16.0, without and with our enhanced single- $\gamma$ background, respectively. Taking into account that there is a difference of two degrees of freedom corresponding to the two fitted oscillation parameters $\sin ^{2} 2 \vartheta_{e \mu}$ and $\Delta m_{41}^{2}$, the statistical significance of the MiniBooNE 


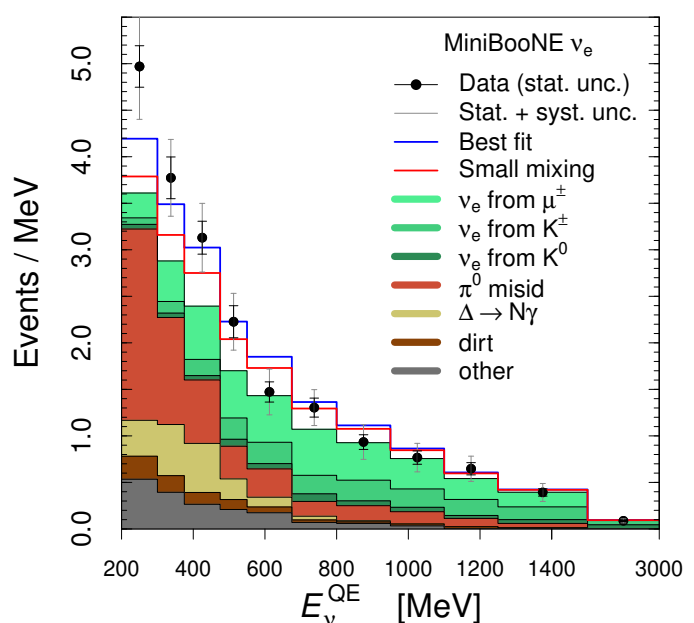

(a)

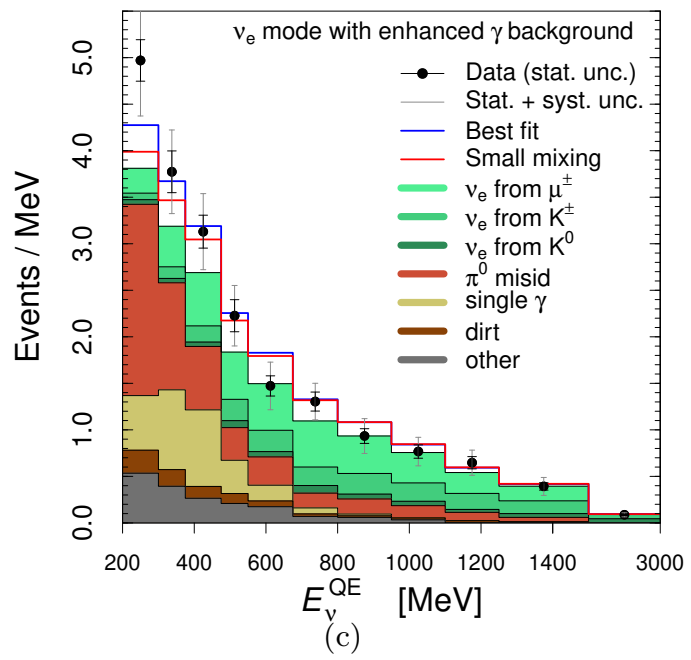

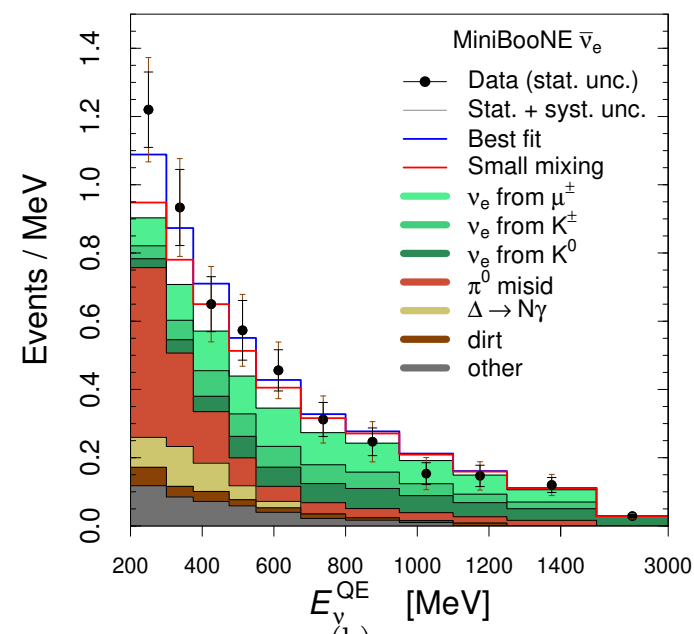

(b)

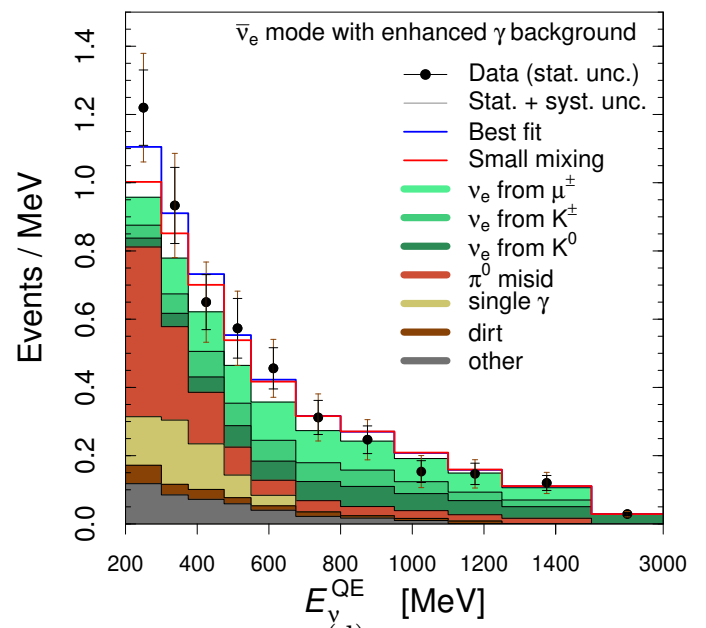

(d)

Figure 1. Comparison of a reproduction of the MiniBooNE event histograms in (a) neutrino and (b) antineutrino mode from refs. [2, 25] with our versions (c) and (d) obtained with the enhanced single- $\gamma$ background due to $A^{1 / 3} \pi^{0}$ FSI in ${ }^{12} \mathrm{C}$, coherent photon emission, incoherent production of higher mass resonances, and incoherent non-resonant nucleon production. The blue and red lines show, respectively, the expectations for neutrino oscillations corresponding to the best fit in table 2 (almost maximal mixing) and the case of small mixing with $\sin ^{2} 2 \vartheta_{e \mu}=2.5 \times 10^{-3}$ and $\Delta m_{41}^{2}=0.8 \mathrm{eV}^{2}$.

indication in favor of oscillation decreases from 5.1 $\sigma$ to $3.6 \sigma$ with the introduction of our enhanced single- $\gamma$ background (the corresponding $\chi^{2}$ probabilities of the background-only fit relative to the best oscillation fit are $2.8 \times 10^{-7}$ and $\left.3.4 \times 10^{-4}\right) .^{2}$

\footnotetext{
${ }^{2}$ In ref. [2] the MiniBooNE collaboration obtained a probability of the background-only fit relative to the best oscillation fit of $6 \times 10^{-7}$, which corresponds to $5.0 \sigma$. The small difference with our result is due to a different analysis of the data performed by the MiniBooNE collaboration with respect to that recommended in their data release [2]. In particular, they considered only the data below $1250 \mathrm{MeV}$ because that upper limit "was chosen by the collaboration before unblinding the data in 2007" [2]. We have no reason to implement this restriction.
} 


\begin{tabular}{|c|cc|cc|cc|}
\hline & $\mathrm{MB}$ & $\widetilde{\mathrm{MB}}$ & LSND+MB & LSND+C & App+MB & App+ $\widetilde{\mathrm{MB}}$ \\
\hline$\chi_{\min }^{2}$ & 22.8 & 20.9 & 29.5 & 21.8 & 81.1 & 72.3 \\
$\mathrm{NDF}$ & 20 & 20 & 25 & 31 & 69 & 75 \\
$\mathrm{GoF}$ & $30 \%$ & $40 \%$ & $24 \%$ & $89 \%$ & $15 \%$ & $57 \%$ \\
$\Delta m_{41}^{2(\mathrm{bf})}$ & 0.0417 & 0.0372 & 0.046 & 0.0398 & 0.58 & 0.692 \\
$\sin ^{2} 2 \vartheta_{e \mu}^{(\mathrm{bf})}$ & 0.98 & 0.98 & 1.00 & 1.00 & 0.0065 & 0.004 \\
\hline
\end{tabular}

Table 2. Minimum $\chi^{2}$, number of degrees of freedom (NDF) and Goodness of Fit (GoF) of the analyses of the data of short-baseline $\stackrel{(-)}{\nu}) \rightarrow \stackrel{(-)}{\nu}$ experiments discussed in the text without (MB) and with $(\widetilde{\mathrm{MB}})$ our enhanced single- $\gamma$ background in MiniBooNE. $\Delta m_{41}^{2(\mathrm{bf})}$ and $\sin ^{2} 2 \vartheta_{e \mu}^{(\mathrm{bf})}$ are the best-fit values of the corresponding oscillation parameters.

From figure 2 one can see that the allowed regions in the $\left(\sin ^{2} 2 \vartheta_{e \mu}, \Delta m_{41}^{2}\right)$ plane change significantly by taking into account our enhanced single- $\gamma$ background. Although the best fit remains at quasi-maximal mixing, there is an extension of the allowed regions towards small values of the mixing parameter $\sin ^{2} 2 \vartheta_{e \mu}$. In particular, the $3 \sigma$ allowed region becomes a band that allows small values of $\sin ^{2} 2 \vartheta_{e \mu}$ of the order of $10^{-3}$ for $\Delta m_{41}^{2} \gtrsim 0.4 \mathrm{eV}^{2}$. This is beneficial, because large active-sterile mixing is disfavored by solar, atmospheric and long-baseline neutrino oscillation data [3-5].

The values of the goodness-of-fit in table 2 show that the fit of MiniBooNE data with the enhanced single- $\gamma$ background is better than the one without, although the difference is small. However, it is more important that the "Small mixing" event histograms in figure 1 show that the small number of signal events resulting from the small mixing $\sin ^{2} 2 \vartheta_{e \mu}=$ $2.5 \times 10^{-3}$ can fit better the low-energy data with our enhanced single- $\gamma$ background. Only the excess in the first bin is not well fitted.

It is interesting to compare the results of our new fit of the MiniBooNE data with the indication of the LSND experiment [26] in favor of short-baseline $\bar{\nu}_{\mu} \rightarrow \bar{\nu}_{e}$ oscillations. Figure 3 shows a comparison of the combined LSND and MiniBooNE allowed regions in the $\left(\sin ^{2} 2 \vartheta_{e \mu}, \Delta m_{41}^{2}\right)$ plane obtained without and with the enhanced single- $\gamma$ background. One can see that the changes are similar to those for MiniBooNE alone (shown in figure 2): there is a clear shift of the allowed regions towards small mixing. In particular, the allowed region with small mixing around $\Delta m_{41}^{2} \approx 2 \mathrm{eV}^{2}$ may be compatible with indications of short-baseline $\bar{\nu}_{e}$ disappearance due to active-sterile neutrino mixing found in reactor experiments $[4,27-32]$.

Finally, we investigated the effects of the analysis of MiniBooNE data with the enhanced single- $\gamma$ background on the global fit of the data of short-baseline $\stackrel{(-)}{\nu} \rightarrow \stackrel{(-)}{\nu}$ oscillation experiments. Besides MiniBooNE and LSND, we considered the data of the BNL-E776 [33], KARMEN [34], NOMAD [35], ICARUS [36] and OPERA [37] experiments, as done in ref. [12]. Figure 4 shows a comparison of the global allowed regions in the $\left(\sin ^{2} 2 \vartheta_{e \mu}, \Delta m_{41}^{2}\right)$ plane obtained without and with the enhanced single- $\gamma$ background in the analysis of MiniBooNE data. One can see that there is again a shift of the allowed regions towards small mixing. Although the shift of the best-fit point towards small mixings is not large, there 


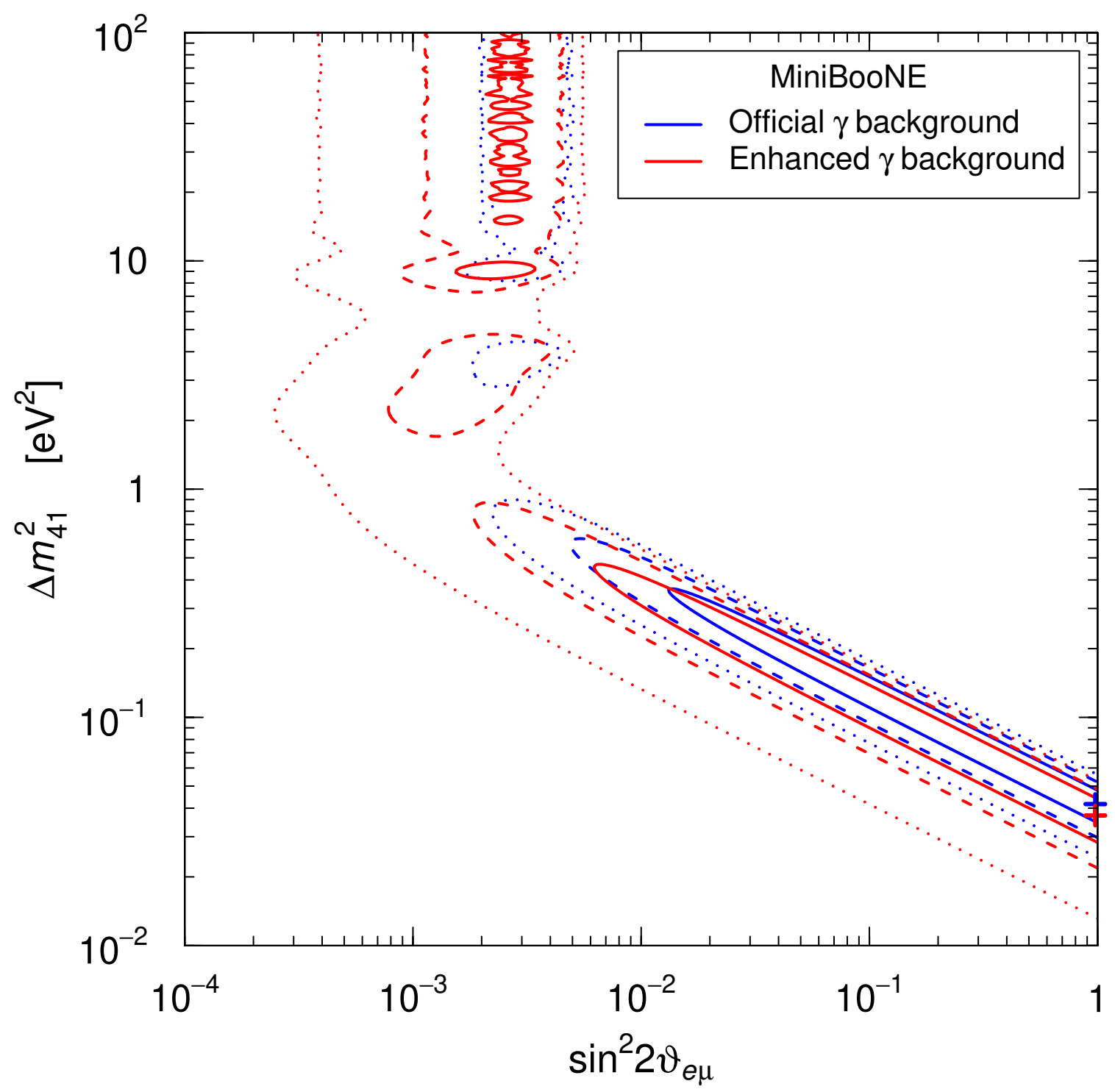

Figure 2. Contours enclosing $1 \sigma$ (solid), $2 \sigma$ (dashed), and $3 \sigma$ (dotted) allowed regions in the $\left(\sin ^{2} 2 \vartheta_{e \mu}, \Delta m_{41}^{2}\right)$ plane obtained from the analysis of MiniBooNE data without (blue) and with (red) our single- $\gamma$ background enhancement.

is a much larger shift of the allowed regions, that reach $\sin ^{2} 2 \vartheta_{e \mu} \approx 6 \times 10^{-4}$ at $3 \sigma$ for $\Delta m_{41}^{2} \approx 2 \mathrm{eV}^{2}$. This shift is beneficial for a decrease of the appearance-disappearance tension in the global fit of short-baseline neutrino oscillation data in terms of $3+1$ active-sterile neutrino mixing [10, 12, 27, 28, 38-49].

For example, the $3 \sigma$ upper limit from disappearance data in figure 7 of ref. [28] is about $\sin ^{2} 2 \vartheta_{e \mu} \lesssim 6 \times 10^{-4}$ at $3 \sigma$ for $\Delta m_{41}^{2} \approx 1.3 \mathrm{eV}^{2}$, that is about the same as the $3 \sigma$ lower limit $\sin ^{2} 2 \vartheta_{e \mu} \gtrsim 6 \times 10^{-4}$ in figure 4 for the same value of $\Delta m_{41}^{2}$. It is clear that there is still a considerable appearance-disappearance tension, but it is significantly smaller than that obtained in ref. [28]. 


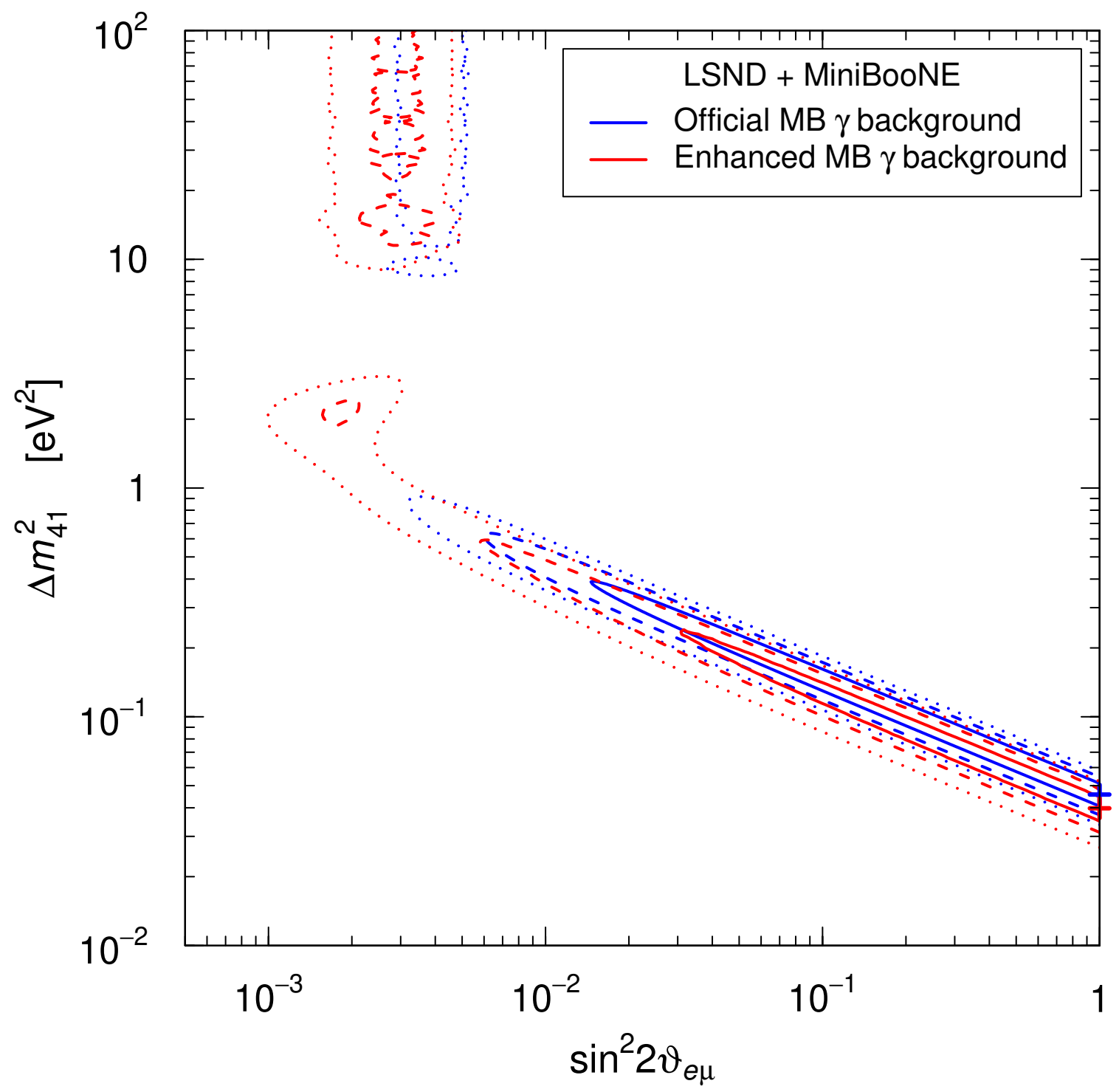

Figure 3. Contours enclosing $1 \sigma$ (solid), $2 \sigma$ (dashed), and $3 \sigma$ (dotted) allowed regions in the $\left(\sin ^{2} 2 \vartheta_{e \mu}, \Delta m_{41}^{2}\right)$ plane obtained from the analysis of LSND and MiniBooNE data without (blue) and with (red) the enhanced single- $\gamma$ background.

The disappearance bound in figure $5 \mathrm{~b}$ of ref. [3] is weaker than that obtained in ref. [28], with an upper limit $\sin ^{2} 2 \vartheta_{e \mu} \lesssim 10^{-3}$ at $3 \sigma$ for $\Delta m_{41}^{2} \approx 1.3 \mathrm{eV}^{2}$, that is compatible with the $3 \sigma$ allowed region in figure 4.

\section{Conclusions}

In conclusion, we have shown that a reassessment of the single- $\gamma$ background from $\Delta^{+/ 0}$ decay in the MiniBooNE experiment taking into account the effect of $A^{1 / 3} \pi^{0}$ FSI proposed in ref. [1] and additional contributions to the single- $\gamma$ background can explain in part the low-energy MiniBooNE excess. In absence of physics beyond the standard three-neutrino 


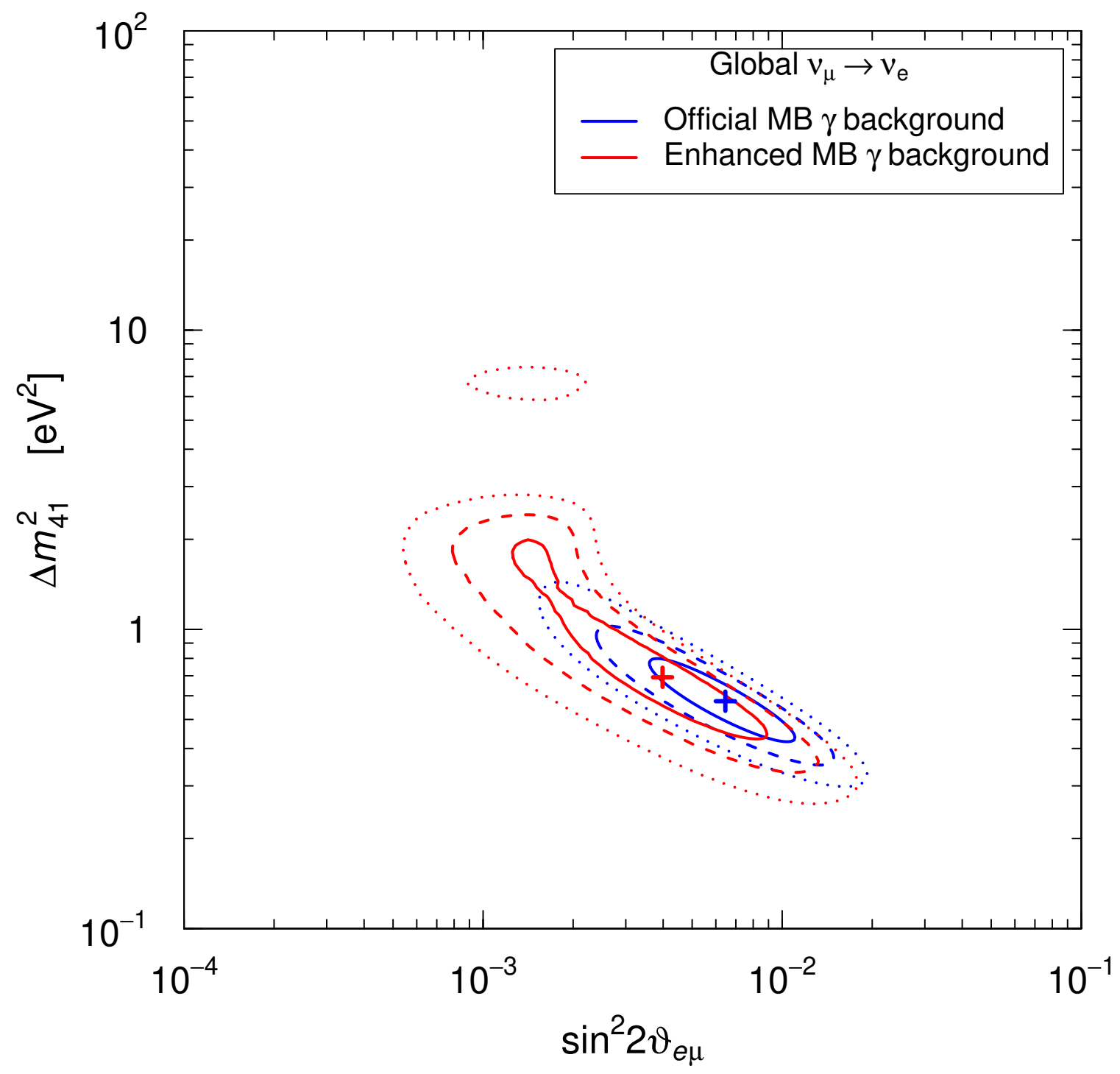

Figure 4. Contours enclosing $1 \sigma$ (solid), $2 \sigma$ (dashed), and $3 \sigma$ (dotted) allowed regions in the $\left(\sin ^{2} 2 \vartheta_{e \mu}, \Delta m_{41}^{2}\right)$ plane obtained from the global analysis of the data of $\stackrel{(-)}{\nu} \rightarrow \stackrel{(-)}{\nu}$ oscillation experiments without (blue) and with (red) the enhanced single- $\gamma$ background in the analysis of MiniBooNE data.

mixing, our enhanced single- $\gamma$ background leads to a better fit of the data, with a goodnessof-fit of $2 \%$, with respect to the standard analysis, that has a goodness-of-fit of $0.02 \%$. However, the MiniBooNE data are still fitted in a better way considering short-baseline $\stackrel{(-)}{\nu} \rightarrow \stackrel{(-)}{\nu}$ oscillations due to active-sterile neutrino mixing, albeit the statistical significance of the indication in favor of oscillation decreases from $5.1 \sigma$ to $3.6 \sigma$. We have shown that in the $3+1$ framework the new analysis of the MiniBooNE data allows smaller values of activesterile neutrino mixing with respect to the standard analysis. This shift towards small active-sterile neutrino mixing is beneficial towards a possible solution of the appearancedisappearance tension in the global fit of short-baseline neutrino oscillation data. 


\section{Acknowledgments}

We would like to thank W.C. Louis and C.A. Ternes for useful discussions. The work of C. Giunti was partially supported by the research grant "The Dark Universe: A Synergic Multimessenger Approach" number 2017X7X85K under the program PRIN 2017 funded by the Ministero dell'Istruzione, Università e della Ricerca (MIUR).

Open Access. This article is distributed under the terms of the Creative Commons Attribution License (CC-BY 4.0), which permits any use, distribution and reproduction in any medium, provided the original author(s) and source are credited.

\section{References}

[1] A. Ioannisian, A Standard Model explanation for the excess of electron-like events in MiniBooNE, arXiv:1909.08571 [INSPIRE].

[2] MiniBooNE collaboration, Significant Excess of ElectronLike Events in the MiniBooNE Short-Baseline Neutrino Experiment, Phys. Rev. Lett. 121 (2018) 221801 [arXiv: 1805.12028] [INSPIRE].

[3] C. Giunti and T. Lasserre, eV-scale Sterile Neutrinos, Ann. Rev. Nucl. Part. Sci. 69 (2019) 163 [arXiv: 1901.08330] [INSPIRE].

[4] A. Diaz, C.A. Argüelles, G.H. Collin, J.M. Conrad and M.H. Shaevitz, Where Are We With Light Sterile Neutrinos?, Phys. Rept. 884 (2020) 1 [arXiv:1906.00045] [INSPIRE].

[5] S. Böser et al., Status of Light Sterile Neutrino Searches, Prog. Part. Nucl. Phys. 111 (2020) 103736 [arXiv: 1906.01739] [INSPIRE].

[6] E. Bertuzzo, S. Jana, P.A.N. Machado and R. Zukanovich Funchal, Dark Neutrino Portal to Explain MiniBooNE excess, Phys. Rev. Lett. 121 (2018) 241801 [arXiv:1807.09877] [INSPIRE].

[7] P. Ballett, S. Pascoli and M. Ross-Lonergan, U(1)' mediated decays of heavy sterile neutrinos in MiniBooNE, Phys. Rev. D 99 (2019) 071701 [arXiv:1808.02915] [INSPIRE].

[8] C.A. Argüelles, M. Hostert and Y.-D. Tsai, Testing New Physics Explanations of the MiniBooNE Anomaly at Neutrino Scattering Experiments, Phys. Rev. Lett. 123 (2019) 261801 [arXiv:1812.08768] [INSPIRE].

[9] P. Coloma, IceCube/DeepCore tests for novel explanations of the MiniBooNE anomaly, Eur. Phys. J. C 79 (2019) 748 [arXiv: 1906.02106] [InSPIRE].

[10] C. Giunti, M. Laveder, Y.F. Li and H.W. Long, Pragmatic View of Short-Baseline Neutrino Oscillations, Phys. Rev. D 88 (2013) 073008 [arXiv: 1308.5288] [InSPIRE].

[11] M. Ericson, M.V. Garzelli, C. Giunti and M. Martini, Assessing the role of nuclear effects in the interpretation of the MiniBooNE low-energy anomaly, Phys. Rev. D 93 (2016) 073008 [arXiv: 1602.01390] [INSPIRE].

[12] S. Gariazzo, C. Giunti, M. Laveder and Y.F. Li, Updated Global 3+1 Analysis of Short-BaseLine Neutrino Oscillations, JHEP 06 (2017) 135 [arXiv:1703.00860] [INSPIRE].

[13] V. Brdar, O. Fischer and A.Y. Smirnov, Model Independent Bounds on the Non-Oscillatory Explanations of the MiniBooNE Excess, arXiv:2007.14411 [INSPIRE]. 
[14] R.J. Hill, On the single photon background to $\nu_{e}$ appearance at MiniBooNE, Phys. Rev. D 84 (2011) 017501 [arXiv: 1002.4215] [INSPIRE].

[15] X. Zhang and B.D. Serot, Can neutrino-induced photon production explain the low energy excess in MiniBooNE?, Phys. Lett. B 719 (2013) 409 [arXiv:1210.3610] [INSPIRE].

[16] E. Wang, L. Álvarez-Ruso and J. Nieves, Single photon events from neutral current interactions at MiniBooNE, Phys. Lett. B 740 (2015) 16 [arXiv:1407.6060] [InSPIRE].

[17] MicroBooNE collaboration, Accelerator-based Short-baseline Neutrino Oscillation Experiments, arXiv:1510.04412 [INSPIRE].

[18] C.M. Ignarra, Sterile Neutrino Searches in MiniBooNE andMicroBooNE, FERMILAB-THESIS-2014-31.

[19] Particle Data Group collaboration, Review of Particle Physics, Phys. Rev. D 98 (2018) 030001 [INSPIRE].

[20] MiniBooNE collaboration, Updated MiniBooNE Neutrino Oscillation Results with Increased Data and New Background Studies, arXiv:2006.16883 [INSPIRE].

[21] B. Krusche et al., Photoproduction of pi0 mesons from nuclei, Eur. Phys. J. A 22 (2004) 277 [nucl-ex/0406002] [INSPIRE].

[22] B. Krusche, Photoproduction of mesons from nuclei: In-medium properties of hadrons, Prog. Part. Nucl. Phys. 55 (2005) 46 [nucl-ex/0411033] [InSPIRE].

[23] B. Krusche et al., Surface and volume effects in the photoabsorption of nuclei, Eur. Phys. J. A 22 (2004) 347 [nucl-ex/0411009] [inSPIRE].

[24] T. Leitner, O. Buss, U. Mosel and L. Álvarez-Ruso, Neutrino scattering with nuclei: Theory of low energy nuclear effects and its applications, PoS NUFACT08 (2008) 009 [arXiv:0809.3986] [INSPIRE].

[25] MiniBooNE collaboration, Improved Search for $\bar{\nu}_{\mu} \rightarrow \bar{\nu}_{e}$ Oscillations in the MiniBooNE Experiment, Phys. Rev. Lett. 110 (2013) 161801 [arXiv:1303.2588] [INSPIRE].

[26] LSND collaboration, Evidence for neutrino oscillations from the observation of $\bar{\nu}_{e}$ appearance in a $\bar{\nu}_{\mu}$ beam, Phys. Rev. D 64 (2001) 112007 [hep-ex/0104049] [INSPIRE].

[27] S. Gariazzo, C. Giunti, M. Laveder and Y.F. Li, Model-independent $\bar{\nu}_{e}$ short-baseline oscillations from reactor spectral ratios, Phys. Lett. B 782 (2018) 13 [arXiv:1801.06467] [INSPIRE].

[28] M. Dentler et al., Updated Global Analysis of Neutrino Oscillations in the Presence of eV-Scale Sterile Neutrinos, JHEP 08 (2018) 010 [arXiv:1803.10661] [INSPIRE].

[29] J.M. Berryman and P. Huber, Reevaluating Reactor Antineutrino Anomalies with Updated Flux Predictions, Phys. Rev. D 101 (2020) 015008 [arXiv:1909.09267] [InSPIRE].

[30] C. Giunti, Y.F. Li and Y.Y. Zhang, KATRIN bound on 3+1 active-sterile neutrino mixing and the reactor antineutrino anomaly, JHEP 05 (2020) 061 [arXiv:1912.12956] [INSPIRE].

[31] C. Giunti, Statistical Significance of Reactor Antineutrino Active-Sterile Oscillations, Phys. Rev. D 101 (2020) 095025 [arXiv: 2004.07577] [INSPIRE].

[32] J.M. Berryman and P. Huber, Sterile Neutrinos and the Global Reactor Antineutrino Dataset, arXiv:2005.01756 [inSPIRE]. 
[33] L. Borodovsky et al., Search for muon-neutrino oscillations $\nu_{\mu} \rightarrow \nu_{e}\left(\nu_{\mu}^{-} \rightarrow \nu_{e}^{-}\right)$in a wide band neutrino beam, Phys. Rev. Lett. 68 (1992) 274 [INSPIRE].

[34] KARMEN collaboration, Upper limits for neutrino oscillations $\nu_{\mu}^{-} \rightarrow \nu_{e}^{-}$from muon decay at rest, Phys. Rev. D 65 (2002) 112001 [hep-ex/0203021] [INSPIRE].

[35] NOMAD collaboration, Search for $\nu_{\mu} \rightarrow \nu_{e}$ oscillations in the NOMAD experiment, Phys. Lett. B 570 (2003) 19 [hep-ex/0306037] [InSPIRE].

[36] ICARUS collaboration, Search for anomalies in the $\nu_{e}$ appearance from a $\nu_{\mu}$ beam, Eur. Phys. J. C 73 (2013) 2599 [arXiv:1307.4699] [INSPIRE].

[37] OPERA collaboration, Search for $\nu_{\mu} \rightarrow \nu_{e}$ oscillations with the OPERA experiment in the CNGS beam, JHEP 07 (2013) 004 [Addendum ibid. 07 (2013) 085] [arXiv:1303.3953] [INSPIRE].

[38] N. Okada and O. Yasuda, A sterile neutrino scenario constrained by experiments and cosmology, Int. J. Mod. Phys. A 12 (1997) 3669 [hep-ph/9606411] [InSPIRE].

[39] S.M. Bilenky, C. Giunti and W. Grimus, Neutrino mass spectrum from the results of neutrino oscillation experiments, Eur. Phys. J. C 1 (1998) 247 [hep-ph/9607372] [INSPIRE].

[40] J. Kopp, M. Maltoni and T. Schwetz, Are There Sterile Neutrinos at the eV Scale?, Phys. Rev. Lett. 107 (2011) 091801 [arXiv:1103.4570] [INSPIRE].

[41] C. Giunti and M. Laveder, 3+1 and 3+2 Sterile Neutrino Fits, Phys. Rev. D 84 (2011) 073008 [arXiv: 1107.1452] [INSPIRE].

[42] C. Giunti and M. Laveder, Status of $3+1$ Neutrino Mixing, Phys. Rev. D 84 (2011) 093006 [arXiv: 1109.4033] [INSPIRE].

[43] C. Giunti and M. Laveder, Implications of 3+1 Short-Baseline Neutrino Oscillations, Phys. Lett. B 706 (2011) 200 [arXiv:1111.1069] [INSPIRE].

[44] J.M. Conrad, C.M. Ignarra, G. Karagiorgi, M.H. Shaevitz and J. Spitz, Sterile Neutrino Fits to Short Baseline Neutrino Oscillation Measurements, Adv. High Energy Phys. 2013 (2013) 163897 [arXiv: 1207.4765 ] [INSPIRE].

[45] M. Archidiacono, N. Fornengo, C. Giunti and A. Melchiorri, Testing 3+1 and 3+2 neutrino mass models with cosmology and short baseline experiments, Phys. Rev. D 86 (2012) 065028 [arXiv:1207.6515] [INSPIRE].

[46] M. Archidiacono, N. Fornengo, C. Giunti, S. Hannestad and A. Melchiorri, Sterile neutrinos: Cosmology versus short-baseline experiments, Phys. Rev. D 87 (2013) 125034 [arXiv: 1302.6720] [INSPIRE].

[47] J. Kopp, P.A.N. Machado, M. Maltoni and T. Schwetz, Sterile Neutrino Oscillations: The Global Picture, JHEP 05 (2013) 050 [arXiv: 1303.3011] [INSPIRE].

[48] S. Gariazzo, C. Giunti, M. Laveder, Y.F. Li and E.M. Zavanin, Light sterile neutrinos, J. Phys. G 43 (2016) 033001 [arXiv: 1507.08204] [INSPIRE].

[49] M. Dentler, A. Hernández-Cabezudo, J. Kopp, M. Maltoni and T. Schwetz, Sterile neutrinos or flux uncertainties? - Status of the reactor anti-neutrino anomaly, JHEP 11 (2017) 099 [arXiv: 1709.04294] [INSPIRE]. 